\title{
Optimization of a Tube Hydroforming Process
}

\author{
Nader Abedrabbo*, Naeem Zafar*, Ron Averill*, Farhang Pourboghrat* and Ranny Sidhuł \\ *Department of Mechanical Engineering, Michigan State University, East Lansing, MI 48824-1226 \\ ‡ Red Cedar Technology, East Lansing, MI 48823
}

\begin{abstract}
An approach is presented to optimize a tube hydroforming process using a Genetic Algorithm (GA) search method. The goal of the study is to maximize formability by identifying the optimal internal hydraulic pressure and feed rate while satisfying the forming limit diagram (FLD). The optimization software HEEDS is used in combination with the nonlinear structural finite element code LS-DYNA to carry out the investigation. In particular, a sub-region of a circular tube blank is formed into a square die. Compared to the best results of a manual optimization procedure, a $55 \%$ increase in expansion was achieved when using the pressure and feed profiles identified by the automated optimization procedure.
\end{abstract}

\section{Introduction}

Aside from the target of cost reduction, industrial enterprises are aiming for optimization of their products regarding weight as well as stability and rigidity. This requires reevaluation of conventional design solutions, manufacturing techniques and material selections in the search for alternative solutions. Such an alternative with interesting technical and economical potential is hydroforming, a method for manufacturing a wide range of complicated hollow components from tubular or sheet blank material by means of water pressure. This method can decrease development times, reduce the number of operation steps, achieve a high precision and undisturbed material structure and promote uniform strength in the component.

Tube hydroforming (THF) technology has drawn increasing attention in the automotive industry because of its enormous advantages over the more traditional processes. These advantages include part consolidation, weight reduction due to improved part design, improved structural strength and stiffness and reduction in the associated tooling and material costs. The range of parts currently being produced or developed using tube hydroforming by the automotive industry continues to grow. These include engine cradles, radiator supports, roof side rails, exhaust instrument support panels [1, 2].

Finite element method (FEM) simulation of the hydroforming process has been proven to be an advantageous tool in assisting automotive designs. $\mathrm{Ni}$ [2] and $\mathrm{Wu}$, et al. [3] simulated engine cradle components, and S.D. Liu [4] simulated rectangular bulging using LS-DYNA [5]. The processes of circular bulging and T-shape formation were simulated by Brewster, et al. [6] using Pam-Stamp.

To reduce defects in THF, the applied internal pressure must be high enough to suppress buckling but low enough so as not to cause tube bursting. In conventional process simulation procedures, a pressure profile and feed rate must be supplied as an input to the finite element program. Based on the results of each finite element simulation, an improved pressure profile and feed rate can be identified based on intuition and experience. But this manual iterative process is very time consuming and tedious, and often does not lead to an optimal solution within a reasonable time. There is a need, therefore, to develop an improved methodology to determine the loading paths (i.e., pressure and axial feeding versus time) required to hydroform a tubular part for a given geometry and material. In this paper, HEEDS [7] in combination with LS-DYNA is shown to optimize the process parameters to determine the best loading paths.

\section{Problem Description}

A square shaped die and a circular tube blank are considered in the present work. Displacement and internal pressure curves sought during the 
optimization are such that the circular tube can expand into a square shaped die to a maximum extent without bursting, buckling, or wrinkling. Considering a point ' $P$ ' on the tube as shown in Fig. 1 , as the tube expands in the die, the distance ' $U$ ' traveled by the point increases.

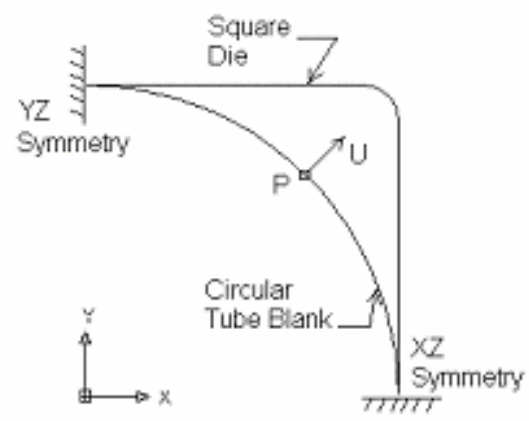

Fig.1. One-eighth model of the tube hydroforming optimization problem.

The optimal rates and values of axial feed and internal pressure are determined so as to maximize the distance ' $U$ ' while avoiding severe thinning and satisfying the forming limit diagram (FLD). The FLD provides information about how much a particular structure can be deformed before necking occurs. Principal strains for each element of the hydroformed tube must lie under the major strain versus minor strain curve of the forming limit diagram to avoid bursting.

The characteristics of the design space associated with the current optimization problem are not known a priori. In this case, it is advisable to employ a combination of global and local search techniques in order to achieve a broad and effective search for an optimal solution. For such problems, HEEDS utilizes a combination of evolutionary, gradient based, and design of experiments search heuristics [7]. Since primarily evolutionary search was used in the present study, a brief description of this search technique is presented in the next section.

\section{Evolutionary Search}

HEEDS (Hierarchical Evolutionary Engineering Design System) is an optimization software package that allows designers to automatically and concurrently explore hundreds of design parameters and their relationships in product and process design scenarios, and intelligently seeks optimal values for parameters that affect performance and cost. HEEDS can be used to improve any engineering system (structural, thermal, fluid, electrical, etc.), including multi-disciplinary problems. It can be applied to parts and processes for any manufacturing process, including stamping, casting, hydroforming, and more.

By automating the iterative manual process normally used to search for designs that simultaneously meet all of the design specifications, engineers can greatly decrease the time required to identify a set of feasible, or even near-optimal, designs prior to building and testing the first prototype. The HEEDS advanced design search algorithms and strategies effectively and efficiently search over a large number of possible design scenarios while performing a relatively small number of design evaluations. HEEDS software operates in a highly parallel computing environment, taking full advantage of powerful and inexpensive computers and networks to modify virtual structure models while simultaneously searching for optimal values of design parameters.

By intelligently coupling global and local search techniques, the HEEDS optimization algorithms are able to find excellent solutions to even the most challenging design problems. Local optimization methods (e.g., nonlinear sequential programming, response surface methods, etc.) are valuable for fine-tuning a design, but not for exploring different design concepts in an effort to identify a much better design. Because the mathematical cost or objective functions associated with many practical design problems are multi-modal (i.e., they have many peaks and valleys) or even discontinuous, the use of global search methods (e.g., parallel genetic algorithms) improves the likelihood of achieving significant design innovation. While global methods search broadly over a large design space, local optimization methods simultaneously focus on promising sub-regions of the design space to identify the best designs in that region.

HEEDS applies several optimization methods simultaneously, allowing each method to take advantage of the best attributes and solutions found from other parallel searches. The multiple semiindependent search processes exchange information about the solution space with each other, helping them jointly to satisfy multiple constraints and objectives. This search method is called a heterogeneous multi-agent approach. This approach quickly identifies design attributes with good 
potential and uses them to focus, improve and accelerate the search for an optimum solution.

\section{Genetic algorithm}

HEEDS employs a Genetic Algorithm (GA) to perform evolutionary search. GAs are particularly useful when the design space is large and complex. The main problem with using a simple GA is the potentially large number of design evaluations required to obtain a set of satisfactory solutions. HEEDS reduces the number of evaluations required to obtain a set of satisfactory solutions by hierarchically decomposing a problem with multiple agents that represent the problem in various ways, while combining efficient local search methods (e.g., response surface methods, nonlinear sequential quadratic programming, and simulated annealing).

A GA is a search procedure that is based on the mechanics of natural selection. Specific knowledge is embedded in a chromosome (or design vector), which represents a possible design with a set of values of all the design variables. The number of choices per design variable determines the fidelity (or resolution) of each design variable. These design variables are the building blocks used to construct a particular design. The GA creates and destroys designs during a process that involves decoding each chromosome, evaluating its satisfaction of constraints and its performance relative to the objectives, then allowing a simulated "natural selection" to determine which designs are eliminated and which survive to generate other derivative designs. Designs that perform well (relative to constraints and objectives) have a higher probability of surviving to influence future designs (their "offspring"). During reproduction, the two genetic operators commonly modeled that produce new chromosomes (or design vectors) are called crossover and mutation.

\section{Crossover}

The crossover operation (sometimes also called "recombination") forms a new solution by combining parts of two existing solutions. A high crossover rate (fraction of population replaced by crossover during one generation of reproduction) will produce many new designs in each generation, but will also have a high probability of disrupting (and potentially losing, at least temporarily) higher-performance designs already found, and requires more evaluations of constraints and objectives in each generation, which are typically the most costly computing operation in the entire problem.

\section{Mutation}

Mutation is a reproduction operation that produces a new solution from a single existing solution, through any of several ways. Mutation can change the value of one design variable or of many simultaneously, and can change them in uniform random ways, or by a normal distribution, for example around the current values of the design variables. Mutation helps maintain diversity and reduces the possibility of premature convergence (the tendency of a set of solutions to come to closely resemble each other, thereby making it difficult for crossover to generate solutions that differ very much from the current set).

A set of co-existing designs defines a population, while successive populations are termed generations. That is, each period during which a set of existing solutions are evaluated then used with natural selection, crossover, and mutation to generate a new set of solutions, is called a generation. A large population typically contains more genetic diversity (i.e., different values of design variables) that typically improves the ultimate results of the GA search. However, the more new individuals created in each generation, the more computer time must be spent evaluating the constraints and objectives of the new individuals, so there is a tradeoff that must be made.

Within each agent, a GA search begins by creating a single initial population, wherein chromosomes (vectors of design variable values) are randomly created. At this point the performance (constraint satisfaction and objective values of each design is evaluated. Biased by the evaluations obtained, the GA uses unary (mutation) and binary (crossover) operators on these designs to create another population. This population probabilistically maintains the previously high performing designs while discarding poorly performing designs. New population members are evaluated, and then additional rounds of generation and selection are performed. This is repeated until satisfactory solution(s) are obtained. Incorporating these processes in a computer routine produces an algorithm that solves problems in a manner reminiscent of natural evolution. Independent GA 
searches in several agents can share information with each other through a user-defined migration process.

\section{Definition of Performance}

The "goodness" of each design is represented with a single scalar value called the performance measure or the objective function. The performance measure is a composite of a number of subsidiary measures, a set of objectives (each of which may be targeted for maximization or minimization) and a set of constraints, for which violations are to be minimized. The constraints enter into the performance according to the penalty method, which gives them no influence so long as they are satisfied, but gives them increasing importance to whatever extent when they fail to be satisfied. Within any single agent, to evaluate the performance measure (or fitness) of a design, the objective and constraint functions are normalized, weighted, and aggregated as follows:

$$
\begin{aligned}
P= & \sum_{i=1}^{\text {Nobjs }}\left(R 1_{i} \frac{f_{o i}}{\left|n_{i}\right|}+R 2_{i}\left(\frac{f_{o i}}{\left|n_{i}\right|}\right)^{2}\right)- \\
& \sum_{i=1}^{\text {Ncons }} C\left(P 1_{i} \frac{\left|f_{C i}-t_{i}\right|}{\left|t_{i}\right|}+P 2_{i}\left(\frac{f_{C i-t_{i}}}{\left|t_{i}\right|}\right)^{2}\right)
\end{aligned}
$$

Where $\mathrm{P}$ is the performance measure, Nobjs is the number of objectives, and Ncons is the number of constraints. $\mathrm{R} 1 \mathrm{i}$ is a constant used to linearly reward a design's performance due to extremizing of the $i_{\text {th }}$ objective function. $\mathrm{R} 2 \mathrm{i}$ is a constant used to quadratically reward a design's performance due to extremizing of the $i_{\text {th }}$ objective function. The $i_{\text {th }}$ objective function $\left(f_{0 \mathrm{i}}\right)$ is normalized by the absolute value of $n_{i} . P 1_{i}$ is a constant used to linearly penalize a design's performance for violating the ith constraint function. $\mathrm{P} 2 \mathrm{i}$ is a constant used to quadratically penalize a design's performance due to the violation of the current constraint function. The ith constraint function $\left(f_{\mathrm{ci}}\right)$ is normalized by the absolute value of its target $t_{i}$. If the constraint is satisfied $\mathrm{C}$ is set to zero; if the constraint is violated $\mathrm{C}$ is set to unity.

\section{Tube Hydroforming Process}

One-eighth part of the tube is considered in the FEM model to reduce the simulation time as shown in Fig. 2. The material model used is the transversely anisotropic elastic plastic model, material 37 in LSDYNA. Material properties obtained from experimental test are shown in Table 1, while Fig. 3 shows the true stress and plastic strain curve used in the simulation.

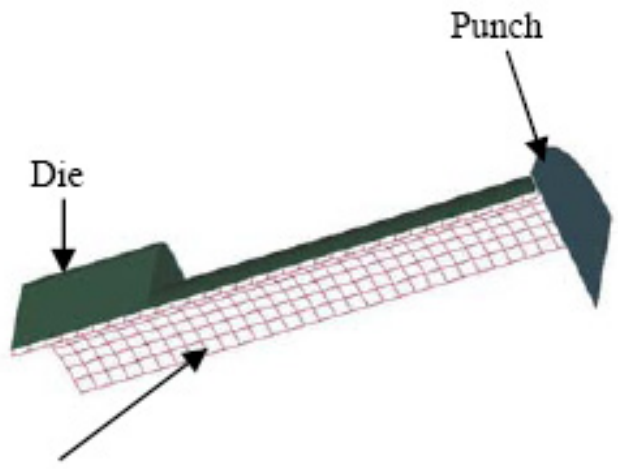

Blank

Fig. 2. One-eighth FEM model of the tube hydroforming process.

Table 1: Material Properties for AA6061-T6.

\begin{tabular}{cccc}
\hline n-value & $\begin{array}{c}\text { Modulus of } \\
\text { Elasticity } \\
\text { E (GPa) }\end{array}$ & $\begin{array}{c}\text { Yield } \\
\text { Strength } \\
(\text { MPa) }\end{array}$ & $\begin{array}{c}\text { Tensile } \\
\text { Strength }\end{array}$ \\
\hline 0.072 & 69 & 235 & 282.77 \\
\hline
\end{tabular}

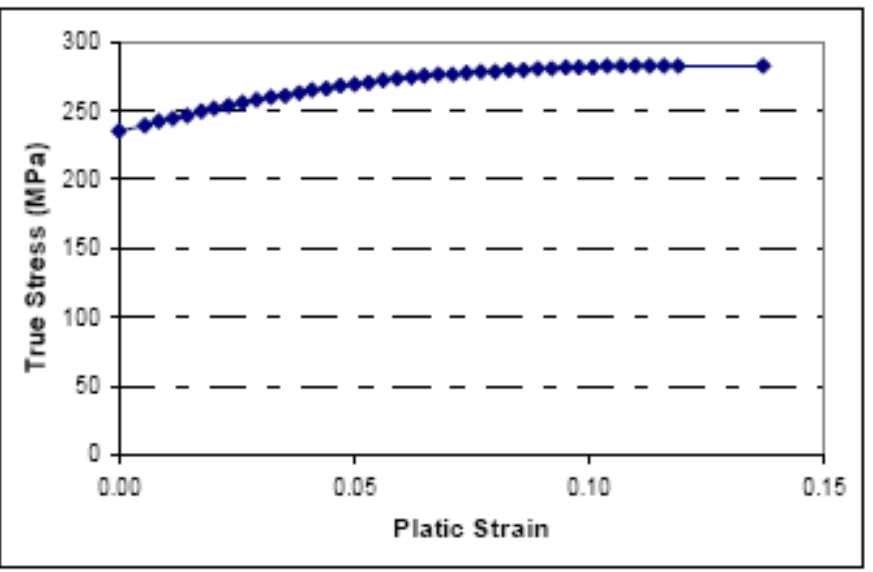

Fig. 3. Experimentally obtained stress-strain data. 


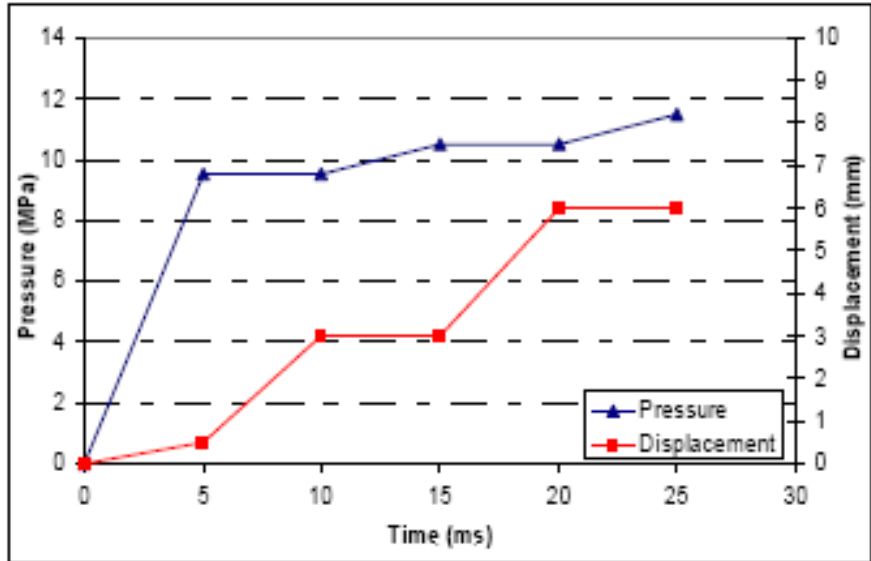

Fig. 4. Internal pressure and axial feed vs. time obtained using a manual iterative process.

\section{Manual Optimization Results}

A large number of manual iterations were performed using LS-DYNA to identify the pressure and axial feed profiles that yielded the maximum expansion of the tube while satisfying the forming limit diagram. One of the best pressures and axial feed profiles found during this manual procedure is illustrated in Fig. 4.

For the pressure and feed rate shown in Fig. 4, the maximum expansion of the tube before failure is illustrated in Fig. 5. The tube expands to a predicted radius of $14.8 \mathrm{~mm}$ before bursting.

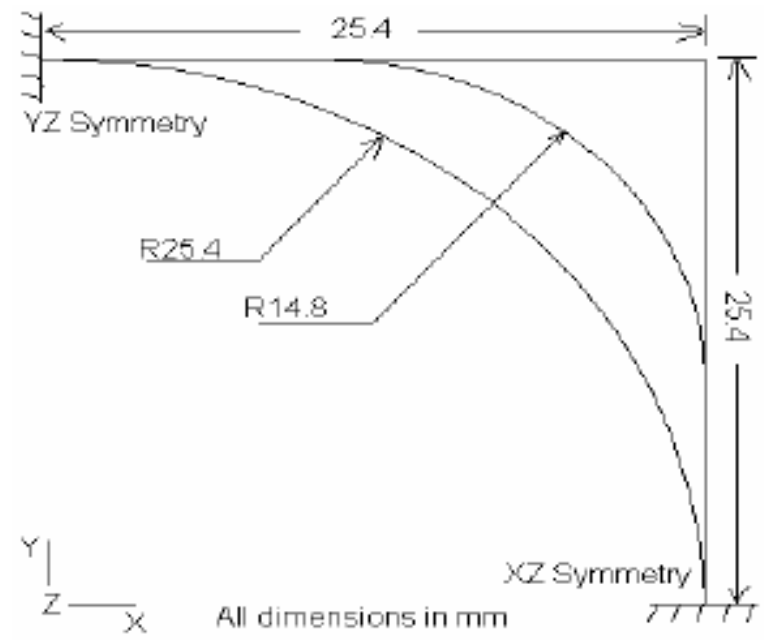

Fig. 5. Maximum radius obtained for the deformed tube before failure using manual optimization.

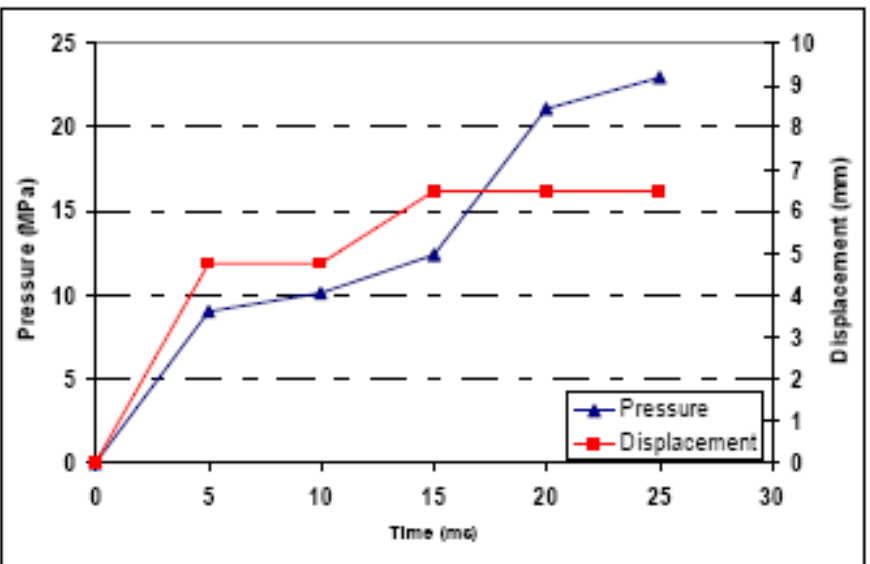

Fig 6. Internal pressure and axial feed obtained from the optimization program HEEDS.

\section{Optimization Results}

HEEDS was used to optimize the fluid pressure and the axial feed profiles. The objective function was to maximize the axial feed in the tube (i.e. to achieve maximum expansion within the square die) while satisfying the constraint on the forming limit diagram (i.e. all strains in the tube fall below the failure limit). HEEDS obtained the displacement and strain component information as output from LSDYNA. Principal strains were calculated and compared with the forming limit diagram acquired for the tube material AA6061-T6. Figure 6 illustrates the best process design values found for the fluid pressure and axial feed rate.

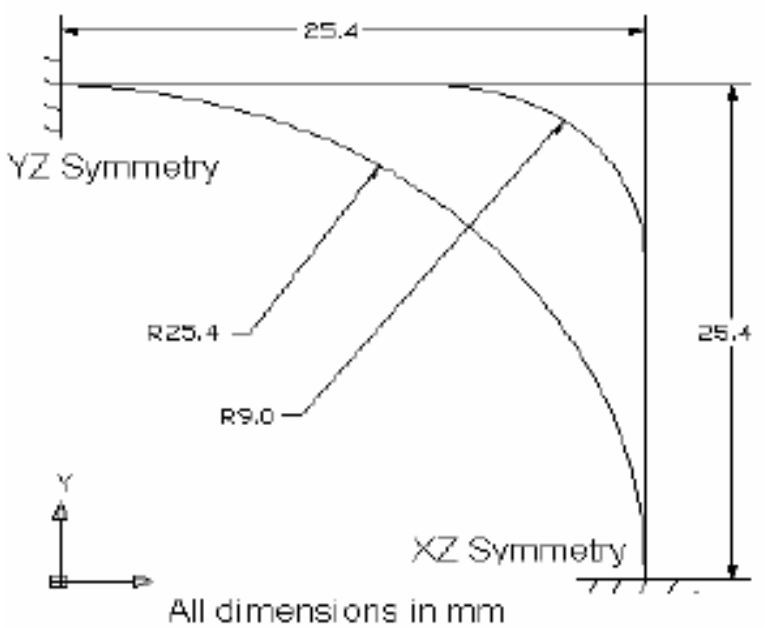

Fig.7. Maximum radius obtained for the deformed tube before failure, as obtained by HEEDS. 


\section{Experimental Validation}

HEEDS was used to optimize the fluid pressure and the axial feed profiles. The objective function was to maximize the axial feed in the tube (i.e. to achieve maximum expansion within the square die) while satisfying the constraint on the forming limit diagram (i.e. all strains in the tube fall below the failure limit). HEEDS obtained the displacement and strain component information as output from LSDYNA. Principal strains were calculated and compared with the forming limit diagram acquired for the tube material AA6061-T6. Figure 6 illustrates the best process design values found for the fluid pressure and axial feed rate.

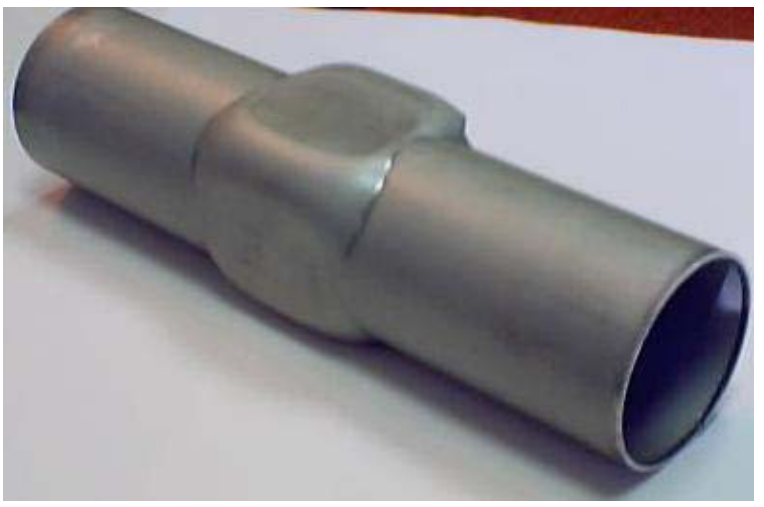

Fig. 8. Experimental result of tube hydroforming process of AA6061-T6.

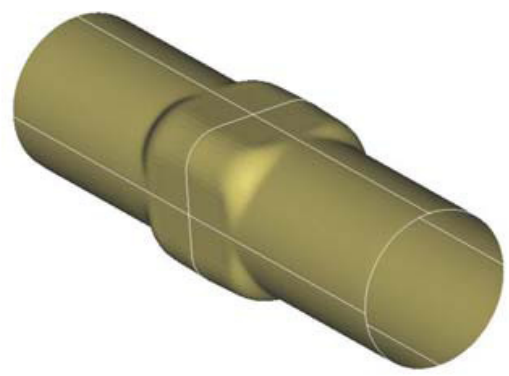

Fig. 9. Numerical result of the tube hydroforming process.

\section{Conclusions}

Hydroforming is an emerging technology that meshes well with the automotive industry's drive to achieve part reduction and more efficient use of material. By using finite element analysis methods in conjunction with automated design optimization procedures, it has been demonstrated here that optimal hydroforming process parameters can be determined very efficiently. In the present study, a $55 \%$ increase in expansion of a circular blank in a square die was achieved compared to manual optimization.

\section{REFERENCES}

1. Ferrier, J., "Hydroforming Paradigms", in Innovations in Hydroforming Technology-1996, Huber and Bauer, Inc.

2. Ni, C.-M., "Stamping and Hydroforming Process Simulation with a 3D Finite Element Code", SAE Technical Paper 940753, 1994.

3. $\mathrm{Wu}, \mathrm{L}$. , and $\mathrm{Yu}, \mathrm{Y}$. , "Computer Simulations of Forming automotive Structural Parts by Hydroforming Process" in Proceedings of Numisheet'96-1996, pp. 324-329.

4. Liu, S. -D., Meuleman D. and K. Thompson, "Analytical and Experimental Examination of Tubular Hydroforming Limits", SAE Technical Paper Series 980449, 1998.

5. Hallquist, J. O., 1998 LS-DYNA Theoretical Manual, Livermore Software Technology Corporation, www.Istc.com.

6. Brewster K., Sutter K., Ahmetoglu M. and Altan T., "Hydro-forming tube", TPG July/August 1996, pp.3440.

7. HEEDS (Hierarchical Evolutionary Engineering Design System) Getting Started Manual, Red Cedar Technology, MI, 48823, USA, www.redcedartech.com.

8. Interlaken Technology Corporation, 8175 Century Blvd., Chaska, MN 55318 USA, www.interlaken.com. 